Journal of Engineering Sciences, Assiut University, Vol. 37, No. 4, pp. 999-1008, July 2009.

\title{
ABSORPTION AND AXIAL RATIO EFFECTS ON THE SCATTERING INTENSITIES OF A SPHEROIDAL PARTICLE ILLUMINATED WITH A PLANE WAVE OR OFF-AXIS GAUSSIAN BEAM
}

\author{
Elsayed Esam M. Khaled, and \\ Elec. Eng. Dept., Eng. Faculty, Assiut University, Assiut, \\ esamk54_2000@hotmail.com
}

\author{
Hany L. Ibrahim \\ Telecom Egypt, Qina, hany_louka@yahoo.com
}

(Received May 28, 2009 Accepted July 5, 2009).

\begin{abstract}
Angular scattering and internal intensities distributions in a lossy dielectric spheroidal particle illuminated with a plane wave or a focused Gaussian beam are investigated. The intensities are calculated using the technique that combining the plane wave spectrum method and the $T$ matrix method. The intensities are calculated as a function of the axial ratio for different spheroid's shape and absorptions. The effects of the incident beam position at or near resonance are also investigated. The angular scattering is much affected by the absorption when the beam is shifted to or outside the edge of the particle. The resonant modes in a lossy oblate particle are much affected by the absorption than those of a lossy prolate particle. The resonant modes having high-Q are sensitive to the axial ratio and absorption changes than the low- $Q$ modes either in a prolate or oblate particle.
\end{abstract}

\section{INTRODUCTION}

Particles in nature such as aerosols content in atmosphere, chemical substances, and rain droplets are nonspherical because they are subjected to tensions, gravity, friction, ....etc. Light scattering by spheroidal particles play important rules in many applications such as blood cells diagnostics, chemical reactions, drugs substance production, particles in semiconductor industry, communication in free space, and nonlinear optical processes in micro- and nano-particles [1-5]. The intensity distributions inside the particles are important for understanding nonlinear optical processes in droplets. The angular scattering and the internal field distributions depend mainly on the particle's shape, and the spatial distribution and frequency of the incident field [5]. Therefore, it is important to study the behaviors of the internal field distributions for particles of different shapes and dielectric properties.

The angular scattering and the internal intensities for different illuminations are calculated for spherical particles $[6,7]$ or for spheroidal particles $[3,5,8,9]$. In the case of a Gaussian beam illumination, the resonances are more easily excited when a beam is focused at or near the edge of the particle than when the beam is focused near the droplet axis [5,6]. For spheroidal particles no studies have been published in the 
literature describing the behaviors of the internal fields inside the particle at or near resonance to show the effects of the shape and absorption on the resonant modes.

For spherical particles the high-Q resonance modes are sensitive to absorption [10]. When a spherical particle is illuminated by a Gaussian beam, focused outside a particle at or near resonant modes the energy in low-order (high-Q) modes is affected more than that in the high-order (low-Q) modes. Does the intensity of a resonant mode in spheroidal particle behave the same as that in spherical particle? What are the factors affecting this behavior? In this paper we intended to answer such questions. The theoretical analysis is outlined in Section 2. Results and discussions are illustrated in Section 3. Conclusions are given in Section 4.

\section{THEORETICAL AND NUMERICAL ANALYSIS}

Figure 1 shows a homogeneous spheroidal particle centered at the origin of a righthanded Cartesian coordinate system $(\mathrm{x}, \mathrm{y}, \mathrm{z})$. A spherical coordinate system $(\mathrm{r}, \theta, \varphi)$ is also shown. The refractive index of the particle is $m$ and that of the surrounding media is unity. The radius of the spheroid along the $x$-axis is $b$ and that along the $z$-axis is $a$. The spheroid is oriented with respect to the Cartesian coordinate system such that its semimajor axis dimension is along the $\mathrm{x}$-axis (an oblate) or along the $\mathrm{z}$-axis (a prolate). The axial ratio is $\rho=a / b$ ( $\rho>1$ for a prolate and $\rho<1$ for an oblate).

The particle is illuminated with either a plane wave polarized in the $\mathrm{x}$-direction or with a lowest order $\left(\mathrm{TEM}_{\mathrm{oo}}\right)$ monochromatic Gaussian beam (it could be of a different shapes) polarized in the $x z$-plane. The incident wave propagates along $z$ direction. The spot size at the beam waist is $\omega_{0}$. The focal point of the beam can be located at an arbitrary point $\left(\mathrm{x}_{\mathrm{o}}, \mathrm{y}_{\mathrm{o}}, \mathrm{z}_{\mathrm{o}}\right)$. The total incident electric field vector $E^{\text {inc }}$ of the beam can be expressed by[6],

$E^{i n c}(x, y, z)=E_{x}^{i n c}(x, y, z) i_{x}+E_{z}^{i n c}(x, y, z) i_{z}$,

where $i_{x}$ and $i_{z}$ are unit vectors in the $x$ and $z$ directions, respectively. The time variation $\mathrm{e}^{-\mathrm{j} \omega \mathrm{t}}$ is omitted.

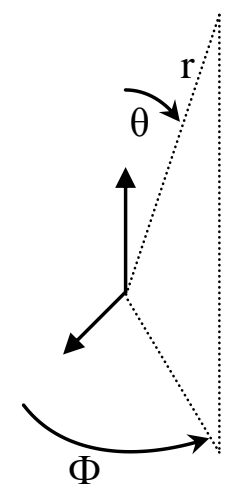

Fig. 1. A spheroidal particle centered at the origin of a Cartesian coordinate system $(x, y, z)$ illuminated with an off-axis focused Gaussian beam of a minimum spot size $w_{o}$.

The radii are $a$, and $b$ along the $z$ - and $x$-axis respectively. The spherical coordinate system is shown 
Mathematical processes are manipulated (details are specified in Reference [6]) to express the incident field in Vector Spherical Harmonics (VSH) as,

$$
E^{i n c}(k r)=P^{2} \sum_{m} \sum_{n} D_{m n}\left[a_{\text {emn }}^{t} M_{\text {emn }}^{1}(k r)+a_{o m n}^{t} M_{\text {omn }}^{1}(k r)+b_{\text {emn }}^{t} N_{\text {emn }}^{1}(k r)+b_{o m n}^{t} N_{o m n}^{1}(k r)\right] \text {, }
$$

where $P$, and $D_{m n}$ are normalization factors. The $m$ and $n$ are integers denoting to the azimuthal mode number and the mode index respectively. The vectors $M^{l}(k r)$, and $N^{l}(k r)$ are the VSH of the first kind, $k=2 \pi / \lambda$ is the wave number; $\lambda$ is the wavelength. The letters $e$ and $o$ stand for even and odd modes respectively. The coefficients $a_{e m n}^{t}$, $a_{\text {omn }}^{t}, b_{\text {emn }}^{t}$, and $b_{\text {omn }}^{t}$ are the expansion coefficients of the incident field. The method can be applied to model any physically realizable beam which can be expressed as a sum of homogeneous plane waves even for the beam that has no mathematical model, e.g. a laboratory non-exact Gaussian beam.

Internal electric fields $E^{\text {int }}$ in a homogeneous particle illuminated with a Gaussian beam using the $T$-matrix method is $[1,5]$,

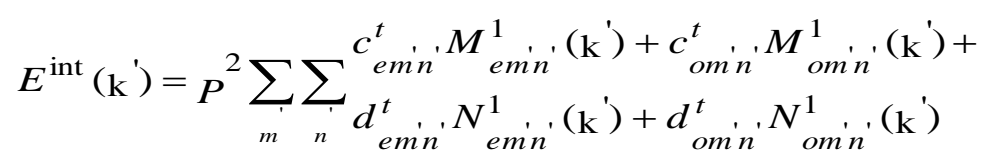

where $k^{\prime}=r k \sqrt{ } \varepsilon_{r} ; \varepsilon_{r}$ is the complex dielectric constant of the scatterer relative to that of the surrounding medium. The $c_{\text {em, }}^{t}, c_{\text {om } n^{\prime}}^{t}, d_{\text {emin }}^{t}$ and $d_{\text {om } n^{\prime}}^{t}$ are the expansion coefficients of the internal electric field. In case of an axisymmetric object $m^{\prime}=m$ and the internal field expansion coefficients for each azimuthal mode, $m$, are given by $[1,5]$,

$$
-i\left[\begin{array}{c}
a_{\sigma}^{t} \\
b_{\sigma}^{t}
\end{array}\right]=[A]\left[\begin{array}{l}
c_{\rho}^{t} \\
d_{\rho}^{t}
\end{array}\right]
$$

where the indices $\sigma$ and $\rho$ are denoting to emn, omn, and emn', omn' respectively. The A-matrix is a block diagonal matrix and each block is a full matrix of dimension $n x n$. Each element in the $A$-matrix is a surface integral over the surface of the scatterer which is calculated numerically. Note that the incident fields coefficients are expanded up to $m$ and $n$ whereas the internal field coefficients are expanded to $m$ and $n$ [1].

\section{RESULTS AND DISCUSSION}

First results are computed to show the effect of the absorption on the angular scattering intensity distributions for three different types of homogeneous prolate spheroids: a lossless prolate of refractive index $\mathrm{m}=1.36$, and lossy prolate spheroids of refractive indices $\mathrm{m}=1.36+\mathrm{i} 0.01$, and $\mathrm{m}=1.36+\mathrm{i} 0.1$. The axial ratio of the prolate is $\rho=1.4$, and its size parameter is $\mathrm{x}=47.3094299$. The spheroid is illuminated with an on-axis Gaussian beam (its focal point is located at the origin) of a minimum spot size $w_{o}=2 \mu m$, and a wavelength $\lambda=1.064 \mu \mathrm{m}$. The beam propagates along the $\mathrm{z}$-direction. The computed angular scattering intensity is shown for the three types of the prolate spheroids in Fig. 2. The same computations are repeated for the same parameters as in Fig. 2 except that 
the beam is shifted to different positions $x_{0}=0.5 \mathrm{~b}=2.8612212 \mu \mathrm{m}, y_{0}=z_{0}=0$, and to $x_{0}=$ $\mathrm{b}=5.7224424 \mu m, y_{0}=z_{0}=0$. The computed results for the shifted beam are shown in Fig. 3 and 4. The calculated results for the three types of the prolate spheroids show that the angular scattering distributions become smoother as the absorption increases. Also more ripples exist for the lossy prolate $(m=1.36+i 0.1)$. The angular scattering intensities changed significantly around the particle as the beam is shifted away from the center.

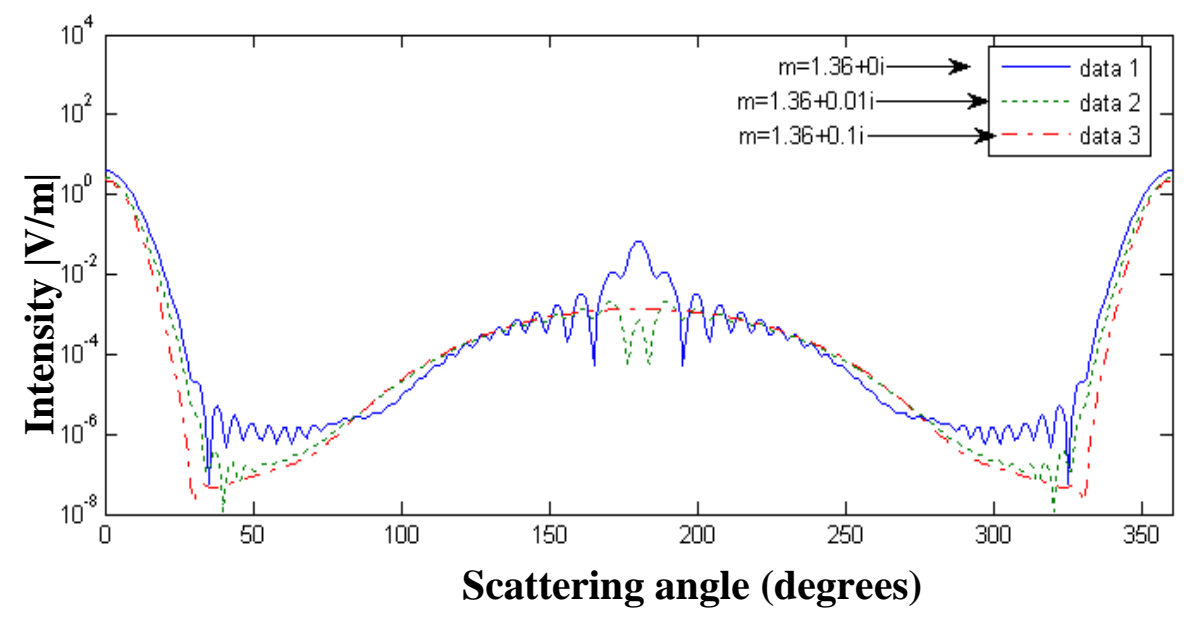

Fig. 2. Angular scattering intensities of a prolate of size parameter $x=47.3094299$ and an axial ratio of $\rho=a / b=1.4$; $\mathrm{a}$ is the semimajor axis along the $\mathrm{z}$-axis and $\mathrm{b}$ is the radius along the $\mathrm{x}$-axis. The prolate is centered at the origin and illuminated with an on-axis

Gaussian beam of a minimum spot size $w_{o}=2 \mu \mathrm{m}$ propagates in the z-direction. The wavelength of the beam is $\lambda=1.064 \mu \mathrm{m}$. The results are computed for a lossless prolate $(\mathrm{m}=1.36)$ and lossy prolate spheroids $(\mathrm{m}=1.36+\mathrm{i} 0.01$, and $\mathrm{m}=1.36+\mathrm{i} 0.1)$.

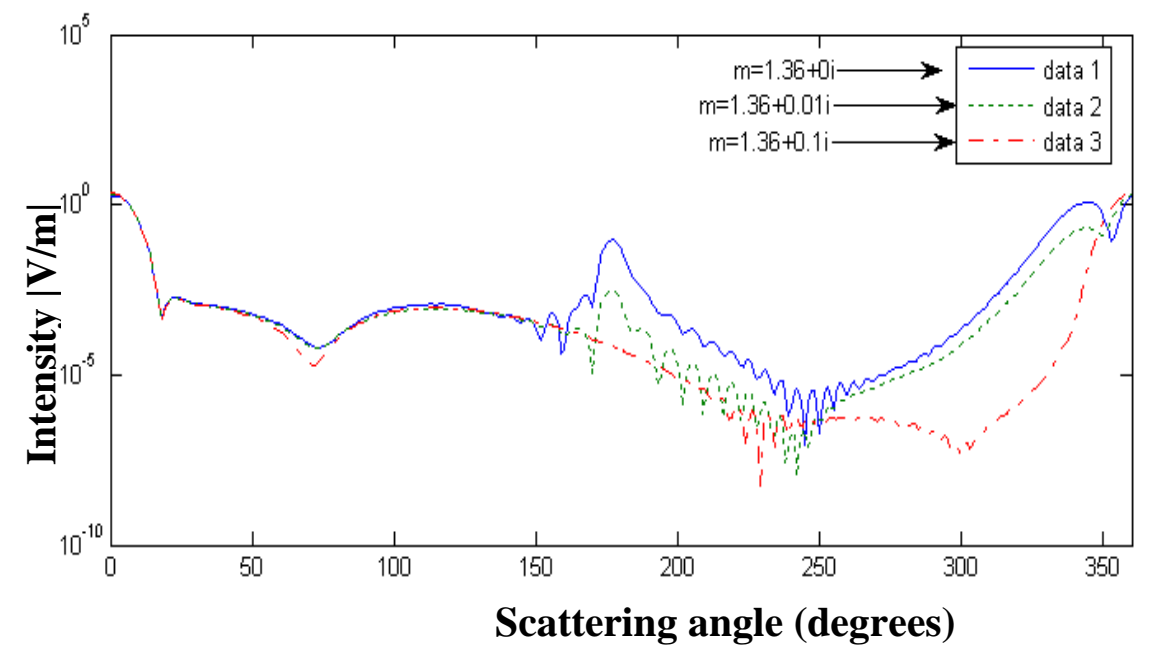

Fig. 3. Angular scattering intensities of a prolate illuminated with a shifted Gaussian beam. The parameters of the prolate and the beam are the same as those in Fig. 2 except that the focal point of the beam is shifted to $x_{0}=0.5 \mathrm{~b}=2.8612212 \mu m, y_{0}=z_{0}=0$. 


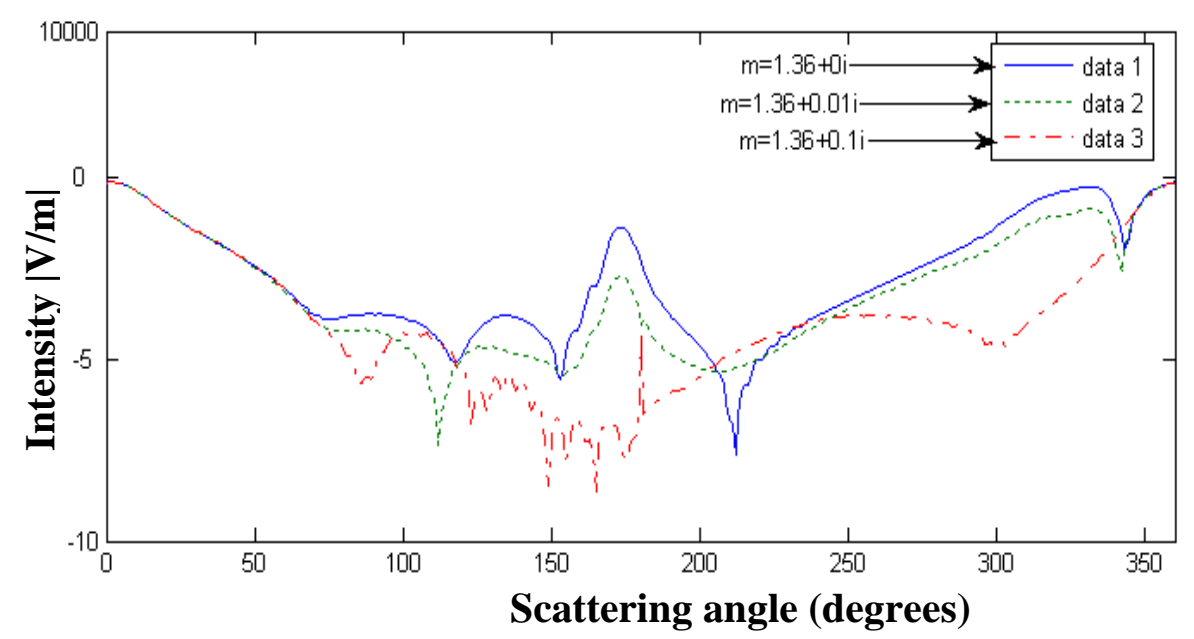

Fig. 4. Angular scattering intensities of a prolate illuminated with a shifted Gaussian beam. The parameters of the prolate and the beam are the same as those in Fig. 2 except that the focal point of the beam is shifted to $x_{0}=\mathrm{b}=5.7224424 \mu \mathrm{m}, y_{0}=z_{0}=0$.

Second the angular scattering intensities are computed for three different types of oblate spheroidal particle of $\mathrm{x}=33.79244991$ and $\rho=0.7143$. The oblate is illuminated with a Gaussian beam of the same parameters as in Fig. 2. The oblate spheroids have refractive indices $\mathrm{m}=1.36, \mathrm{~m}=1.36+\mathrm{i} 0.01$, and $\mathrm{m}=1.36+\mathrm{i} 0.1$ The angular scattering distributions are computed for different values of the beam shifts similar to those shown in Figs. 2-4. The corresponding results are illustrated in Figs. 57.

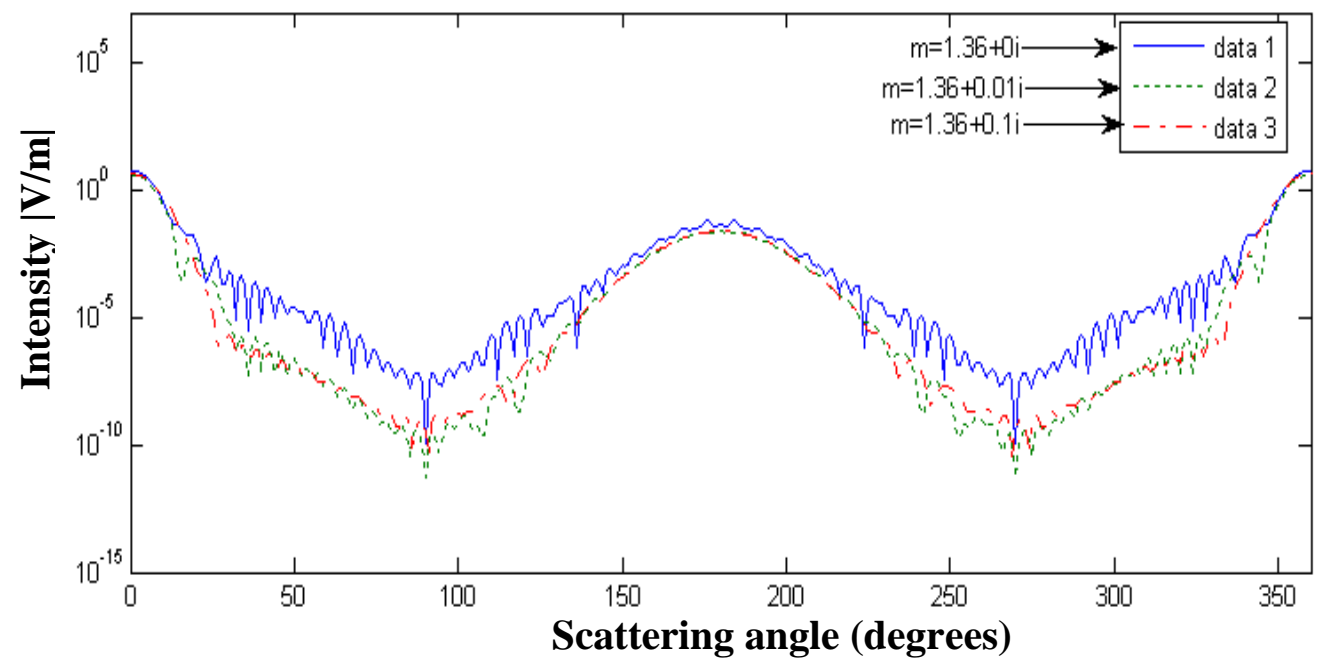

Fig. 5. Angular scattering intensities of an oblate spheroid of size parameter $\mathrm{x}=33.79244991$ and an axial ratio $\rho=a / b=0.7143$; $\mathrm{a}$ is radius along the $\mathrm{z}$-axis, and $\mathrm{b}$ is the semimajor radius along the $\mathrm{x}$-axis. The oblate is centered at the origin and illuminated with an on-axis Gaussian beam of the same parameter as those in Fig. 2.

The results are shown for different oblate types; lossless $(m=1.36)$, and lossy $(\mathrm{m}=1.36+\mathrm{i} 0.01$ and $\mathrm{m}=1.36+\mathrm{i} 0.1)$. 


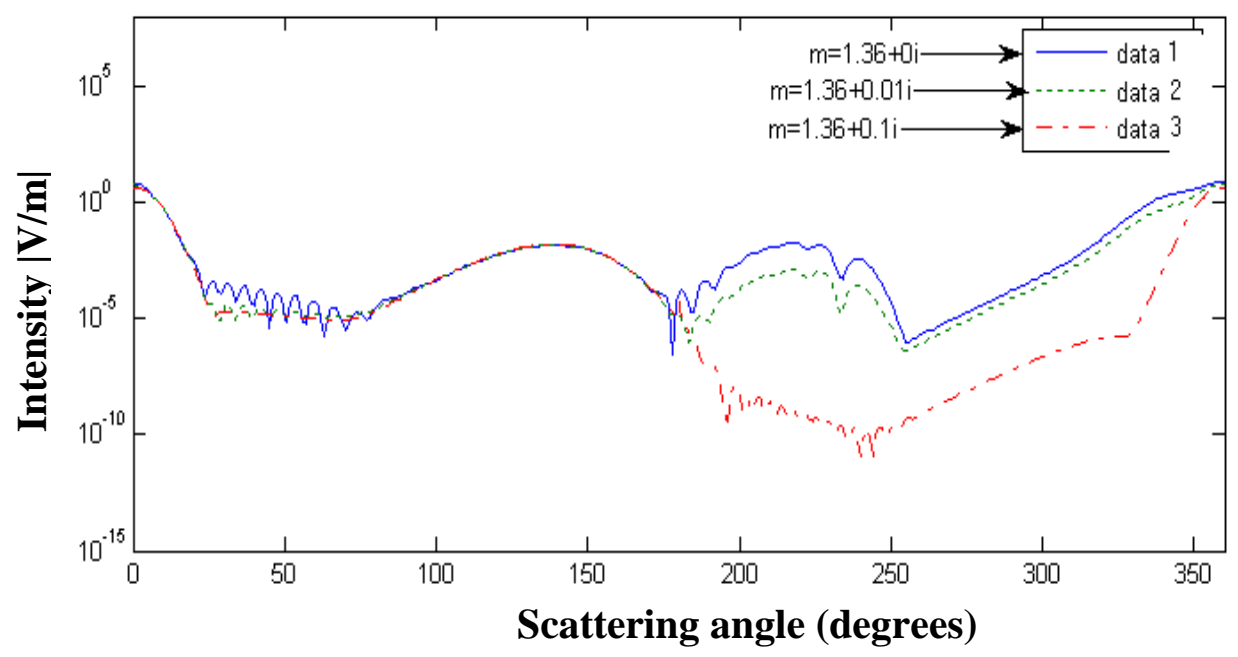

Fig. 6. Angular scattering intensities of an oblate spheroid illuminated with an off-axis Gaussian beam. All the parameters of the oblate and the beam are the same as those in Fig. 5 except that the beam is shifted to $x_{0}=0.5 \mathrm{~b}=4.0057097 \mu m, y_{0}=z_{0}=0$.

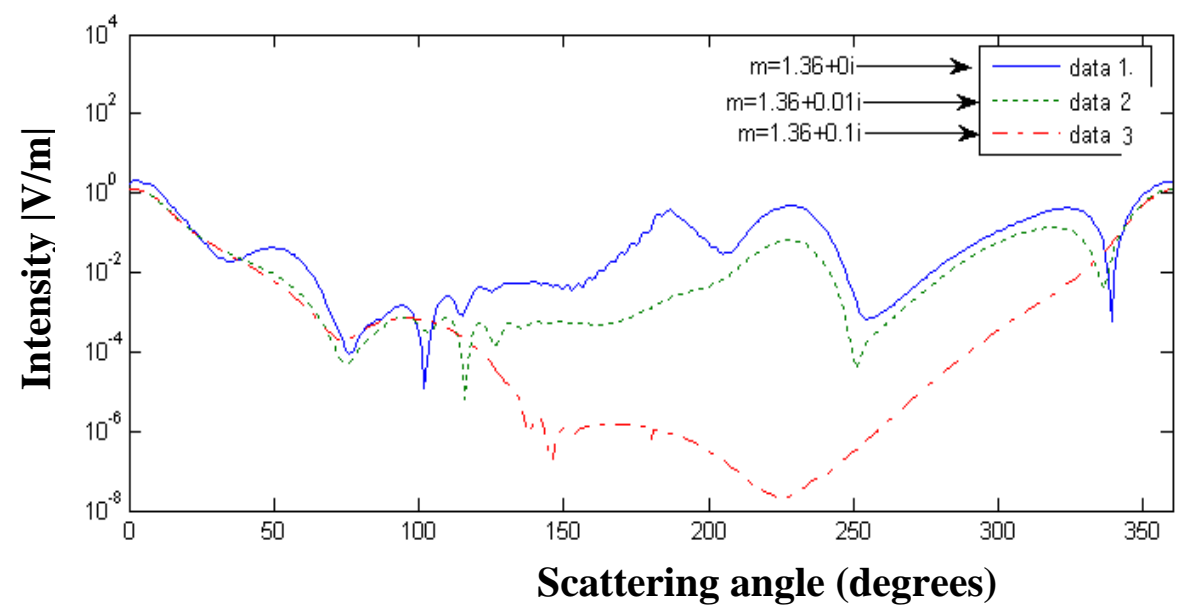

Fig. 7. Angular scattering intensities of an oblate spheroid illuminated with an off-axis Gaussian beam. All the parameters are the same as those illustrated in Fig. 5 except that the beam is shifted to $x_{0}=\mathrm{b}=8.0114194 \mu m, y_{0}=z_{0}=0$.

Figures 2 and 5 show more pronounced ripples around sides of the oblate than those for the prolate case. Also for an oblate particle illuminated with an on-axis beam the ripples are filtered out as the absorption increases. The figures 3, 4, 6, and 7 show that if the beam is shifted to the surface of the scatterer the angular scattering intensities behave differently as the absorption of the spheroid increases. For a lossy oblate with an off-axis illumination the angular scattering is very low around the back scattering direction relative to the other directions.

The behavior of the intenal intensities in a lossless and a lossy spheroid particle as a function of the axial ratio is investigated. A spherical particle of a refractive index $\mathrm{m}=1.36+\mathrm{i} 0$ centered at the origin is considered. The particle is 
illuminated with a plane wave of $\lambda=1.064 \mu \mathrm{m}$ propagating in the $\mathrm{z}$-direction. The size parameter of particle is $\mathrm{x}=47.3094299$ which corresponding to $\mathrm{TE}_{58,1}$ resonant mode. The summation of the magnitude of the internal electric field expansion coefficients over the azimuthal modes $m, \sum_{m}\left|c_{e m n^{\prime}}\right|+\left|c_{o m n^{\prime}}\right|$, is computed. Then the axial ratio is increased and decreased gradually with the same equivalent spherical volume that is equals to the volume of the spherical resonant size parameter. The size parameter of the spheroid at each value of $\rho$ is calculated using the relation $\mathrm{x}_{\text {spheroid }}=\mathrm{x}_{\text {sphere }}(\mathrm{a} / \mathrm{b})^{2 / 3}$. The results are computed for different values of refractive index; lossless spheroid $\mathrm{m}=1.36$, lossy spheroids with $\mathrm{m}=1.36+\mathrm{i} 0.0001$ and $\mathrm{m}=1.36+\mathrm{i} 0.001$. The computed results are illustrated in Fig. 8. The results show peaks at different values of axial ration. Those peaks are corresponding to certain resonant modes.

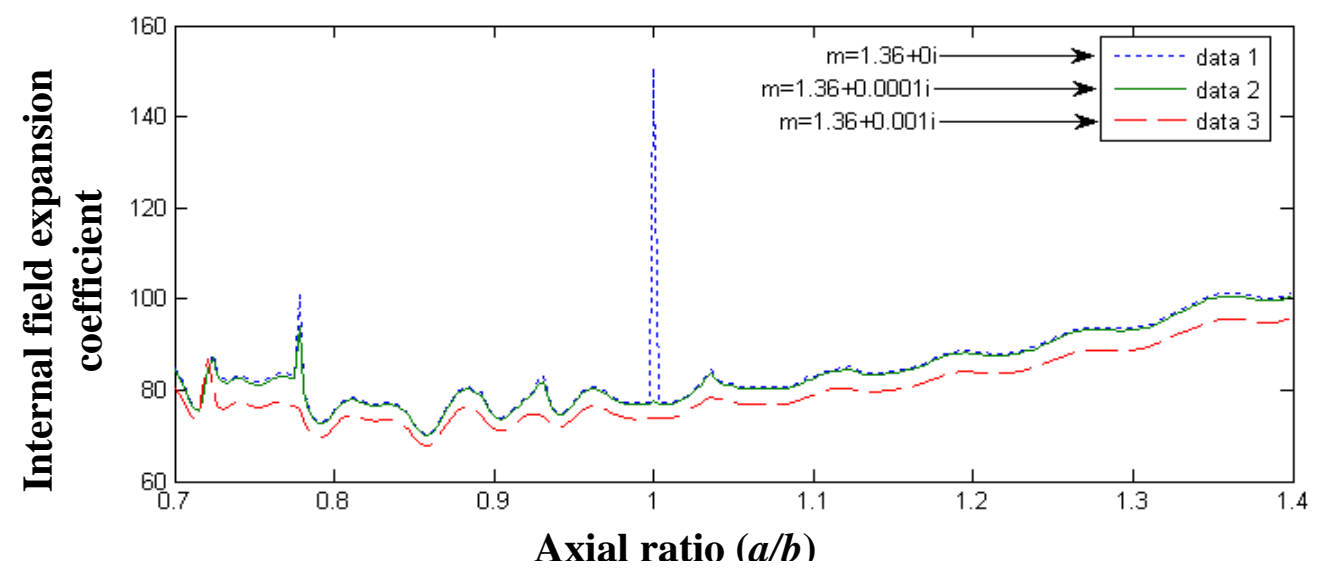

Fig. 8 Summation of the amplitude of the internal field expansion coefficient corresponding to the $\mathrm{TE}_{58,1}$ resonant mode over the azimuthal modes $\mathrm{m}$, that is $\Sigma_{\mathrm{m}}$ $\left|c_{e m n}\right|+\left|c_{\text {omn }}\right|$ as a function of an axial ratio $\rho=a / b$. The size parameter $x=47.3094299$ is corresponding to $\mathrm{TE}_{58,1}$ resonant mode for the spherical particle. The particle is illuminated with a plane wave of a wavelength $\lambda=1.064 \mu \mathrm{m}$, polarized in the $\mathrm{x}-$ direction, and propagates in z-direction. The results are shown for different values of the refractive index $\mathrm{m}$.

The computations illustrated by Fig. 8 are repeated with the same particle's parameters but with an off-axis Gaussian beam illumination. The parameters of the beam are the same as those in Fig. 2 except that the beam is shifted outside the particle to $x_{o}=11.098098 \mu \mathrm{m}, y_{o}=z_{o}=0$. Note that the shift is sufficiently far outside the particle to excite resonant modes. The calculated results are shown in Fig. 9.

Figures 8 and 9 illustrate that the resonance in spheroidal particle is sensitive to the absorption (less than the absorption effect in resonant spherical particle). High-Q modes are more sensitive to the absorption than the low-Q modes. This is applicable to any resonance at any axial ratio. In oblate spheroids the resonant modes are more sensitive to the absorption since distinctive resonant modes can be excited [5]. 


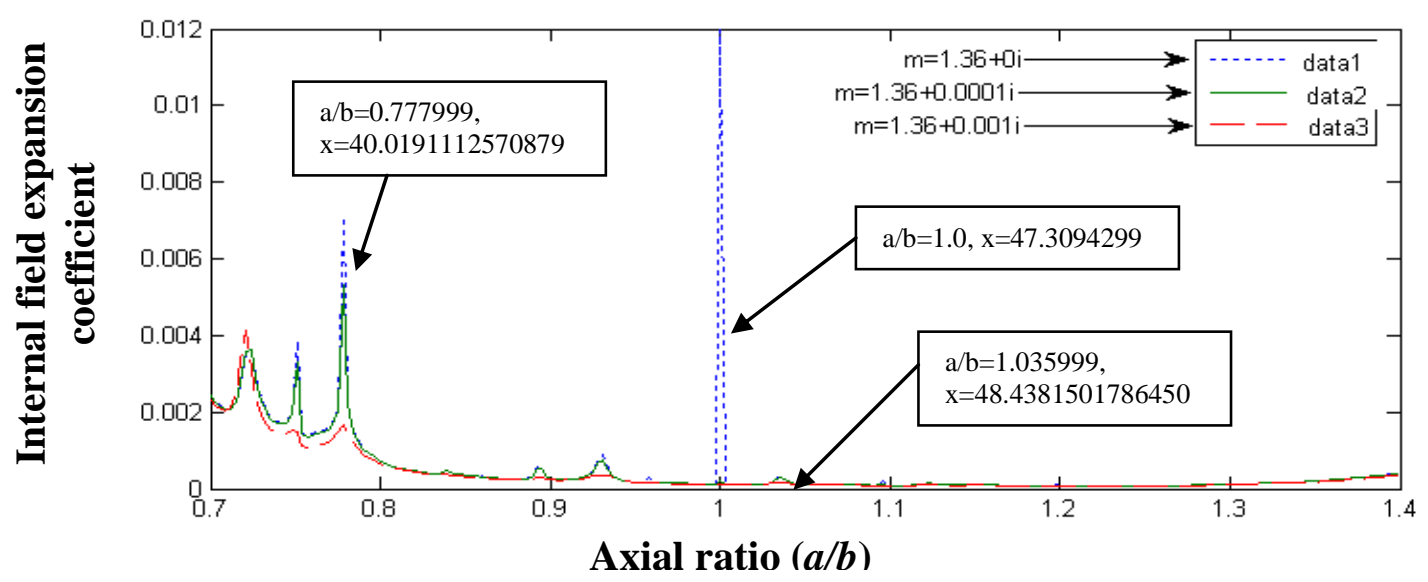

Fig. 9 The summation of the amplitude of the internal field expansion coefficients corresponding to the $\mathrm{TE}_{58,1}$ mode over the azimuthal modes $\Sigma_{\mathrm{m}}\left|\mathrm{c}_{\mathrm{emn}}\right|+\left|\mathrm{c}_{\mathrm{omn}}\right|$ as a function of an axial ratio $\rho=a / b$. The spherical particle size parameter is $x=47.3094299$.

The particle is illuminated with an off-axis Gaussian beam of a wavelength $\lambda=1.064$

$\mu \mathrm{m}$ and minimum spot size $\mathrm{w}_{\mathrm{o}}=2 \mu \mathrm{m}$. The beam is shifted to $x_{o}=11.098098 \mu \mathrm{m}$, $y_{o}=z_{o}=0$ and propagates in z-direction. The results are shown for different values of the refractive index $\mathrm{m},\left(\mathrm{m}=1.36, \mathrm{~m}=1.36+\mathrm{i} 10^{-4}\right.$, and $\left.\mathrm{m}=1.36+\mathrm{i} 10^{-3}\right)$.

\section{CONCLUSIONS}

The angular scattering intensity distributions and the internal intensities are calculated for lossless and lossy spheroidal prolate and oblate particles illuminated with a focused shifted Gaussian beam. As the beam is shifted outside the particle the angular scattering becomes more sensitive to the absorption. As the absorption increases the high-Q modes can be maintained if the beam is shifted farther away from the surface of the spheroid. In case of oblate spheroids the high-Q modes are much affected by the axial ratio and the absorption changes than that in the case of spherical particle.

\section{REFERENCES}

[1] P. W. Barber and S. C. Hill, Light Scattering by Particles: Computational Methods (World Scientific, Singapore, 1990.

[2] J. P. Barton and D. R. Alexander, "Electromagnetic fields for an irregularly shaped, near spherical particle illuminated by a focused laser beam," J. Appl. Phys. 69, 7973-7986, (1991).

[3] J. P. Barton, "Internal and near-surface electromagnetic fields for a spheroidal particle with arbitrary illumination", Appl. Opt. 34, 5542-5551 (1995).

[4] E. E. M. Khaled, "Scattering of an on-axis arbitrarily-shaped focused electromagnetic beam by an elongated spheroidal object," Proceeding of the twenty-sixth national radio science conference (NRSC'26, 2009) sponsored by URSI and IEEE Egypt section, Faculty of Engineering, Future University, Cairo, Egypt, March. 17-19, 2009. 
[5] E. E. M. Khaled, and H. Louka, "Scattered and Internal Intensities of a Homogeneous Dielectric Spheroidal Object Illuminated with an Arbitrary Focused Off-Axis Electromagnetic Beam," accepted for publication in the Faculty of Engineering Scientific Journal, Assiut University, July 2009.

[6] E. E. M. Khaled, S. C. Hill, and P. W. Barber, "Scattered and internal intensity of a sphere illuminated with a Gaussian beam," IEEE Trans. Antennas Propag. 41, 1-9 (1993).

[7] J. P. Barton, D. R. Alexander, and S. A. Schaub," Internal fields of a spherical particle illuminated by a tightly focused laser beam: focal point positioning effects at resonance," J. Appl. Phys. 65, 2900-2906 (1989).

[8] M. I. Mishchenko, L. D. Travis, and A. A. Lacis, Scattering, Absorption, and Emission of Light by Small Particles. NASA Goddard Institute for Space Studies, NewYork, 2005.

[9] Y. Han, and Z. Wu, "Scattering of a spheroidal particle illuminated by a Gaussian beam," Appl. Opt., 15, 40, 2001.

[10] E. E. M. Khaled, S. C. Hill, and P. W. Barber, "Internal electric energy in a spherical particle illuminated with a plane wave or off-axis Gaussian beam," Appl. Opt. 33, 524-532 (1994). 
تأثيرات الأمتصاص ومعامل الأستطالة على شدة المجالات المشتتة لجسيم

\section{بيضاوي مسلط عليه موجة مستوية أو شعاع جاوسين ذو أزاحة}

السيد عصام محمد خالد

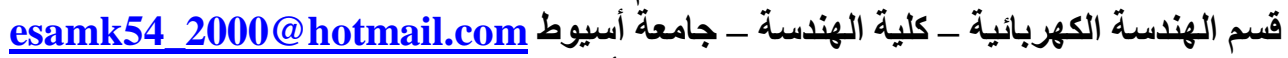
هاني لوقا أبراهيم أبيوط

\section{hany_louka@yahoo.com المصرية للأتصالات ـ متاني لونا}

تم حساب ودراسة شدة المجالات للتشتت الزاوي وأيضاً شدة المجالات داخل جسيم بيضاوى متجانس

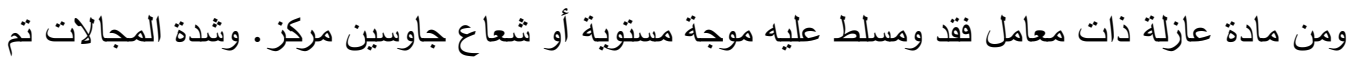

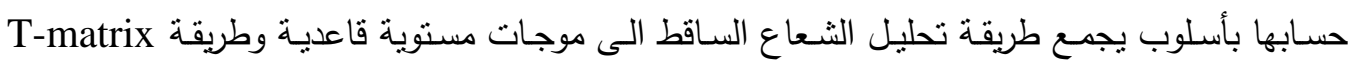
لحساب شدة المجالات داخل وخارج الجسيم والتى تم حسابها كدالة في معمل أستطالة الجسيم لأجسام بيضاوية مختلفة الثكل (prolate and oblate) ومختلفة معامل الأمتصاص. وأيضاً نم دراسة تأثير موضع وأزاحة الثعاع المسلط بالنسبة للجسيم عند أو قريب من حالات الرنين.

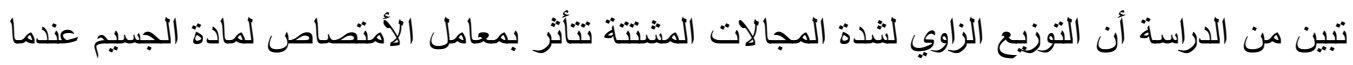

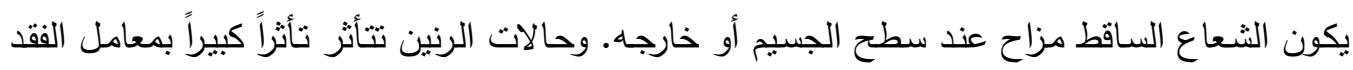

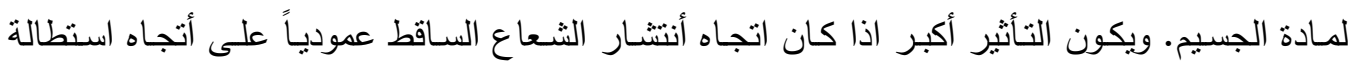
الجسيم (Oblate) عنه اذا كان أتجاه انتشار الثعاع موازياً لأتجاه أسنطالة الجسيم (Prolate).

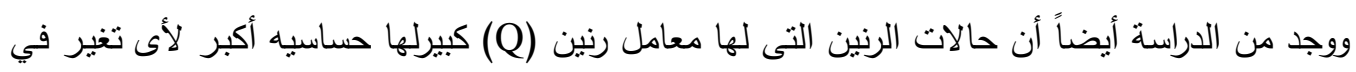

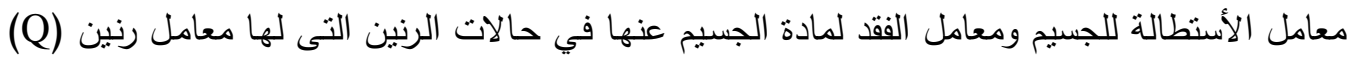
أصغر وذلك في كلا نوعي الجسيم البيضاوي. 